\title{
Diseño metodológico de consignas para atención de fallas no destructivas en subestaciones eléctricas
}

*M.Sc. - Profesor - Facultad de Ingeniería Eléctrica Universidad Tecnológica de Pereira - Pereira, Colombia. e-mail:jmora@utp.edu.co

**Ph.D. Director del Grupo de Sistemas de Energía Eléctrica -GISEL - Profesor - Escuela de Ingeniería Eléctrica - Universidad Industrial de Santander Bucaramanga, Colombia. e-mail:gilberto@uis.edu.co

$\star \star \star$ M.Sc. Investigador Universidad Industrial de Santander Bucaramanga, Colombia. e-mail:jariza@uis.edu.co
Fecha de recepción: Septiembre 25 de 2004 Fecha de aprobación: Junio 10 de 2005

\author{
Juan José Mora Flórez.* \\ Gilberto Carrillo Caicedo** \\ Jorge Olmedo Ariza Castillo** \\ Je Olmedo Ariza Castillo*n.
}

\section{RESUMEN}

Las fallas en las subestaciones pueden ocasionar pérdida de la continuidad del suministro de energía eléctrica desde las fuentes a las cargas. Por este motivo, es necesario el planteamiento de estrategias y la elaboración de procedimientos para atender estas circunstancias en el menor tiempo posible.

En este articulo, se presenta la metodología utilizada para desarrollar las estrategias de atención de las fallas no destructivas en los equipos de patio, de servicios auxiliares, de control, de protección, de medida, de registro de fallas y de comunicaciones de subestaciones de 
transmisión bajo responsabilidad de ISA CTE Oriente. El objeto de estas estrategias es reducir el tiempo de indisponibilidad de los equipos, y con ello, los índices de energía no suministrada por el sistema de potencia.

Palabras clave: Subestaciones, fallas, consignas bajo falla, equipo de potencia.

\section{ABSTRACT}

The continuity of the electrical service from the source to the load is generally interrupted by faults at electrical substations. Because of that, the development of special procedures to restore the faulted element in a short time period is considered necessary.

This paper presents the methodology followed to develop strategies useful attend non destructive faults in power equipment; substation, control, relaying, measurement, fault recorders and communication services in transmission substations from CTE Oriente. The goal of these strategies is to reduce the unavailability time of power equipment and as a consequence the reduction of the non-supplied energy index of the power system.

Key words: Electrical substations, faults, restoration strategies, power equipment.

\section{INTRODUCCIÓN}

La calidad de la energía eléctrica, desde la perspectiva técnica, valora la continuidad y la forma de las señales de tensión y de corriente eléctrica, evaluando así el servicio y el suministro que las empresas proporcionan al usuario [1] [2]. La continuidad del suministro involucra aspectos relacionados con la atención de fallas en equipos con el fin de garantizar su disponibilidad. La atención de fallas tiene como objetivo la reducción del tiempo en que los elementos del sistema están indisponibles [3]. Para conseguir una reducción efectiva del tiempo de indisponibilidad se busca la interacción y la sinergia entre: a) Los programas de mantenimiento, b) Las estrategias de atención de fallas, en casos de indisponibilidad de los equipos y, c) La capacitación de los operadores de la subestación.

Dada la importancia que tienen las subestaciones de transmisión en la operación del sistema de potencia, el principal transportador de energía de Colombia, Interconexión Eléctrica (ISA) y la Universidad Industrial de Santander (UIS), desarrollaron estrategias de restauración de equipos en caso de fallas no destructivas. Mediante estas estrategias se pretende minimizar el tiempo de contingencia, así como las consecuencias para el funcionamiento de la subestación. En [2] y [3] se presenta un análisis teórico previo a este trabajo, del procedimiento para la selección de equipos críticos en una subestación de transmisión de energía.

En este artículo se describe la metodología para elaborar los procedimientos de atención de fallas no destructivas en los equipos y sistemas de una subestación de transmisión. Se presenta adicionalmente la aplicación de los procedimientos en los equipos de las subestaciones de ISA, regional CTE Oriente.

\section{FALLAS EN LAS SUBESTACIONES DE TRANSMISIÓN}

Los sistemas y equipos de las subestaciones están diseñados para que funcionen manteniendo una alta confiabilidad. Mo obstante, una falla puede ocurrir en cualquier punto del sistema o equipo, causando mal funcionamiento de una parte o de toda la subestación.

Las fallas, según su severidad, se clasifican en destructivas y no destructivas. Las fallas destructivas implican pérdida total del equipo afectado y en consecuencia la restitución del mismo, ocasionando la disminución en la confiabilidad, flexibilidad y seguridad del sistema de potencia. Las fallas no destructivas son aquellas en las cuales el operador y los grupos de 
mantenimiento de la subestación, pueden realizar labores de reparación sobre el equipo afectado. Estas fallas implican la disminución de la capacidad operativa y de control de las subestaciones.

Las maniobras de funcionamiento normal de los equipos de una subestación están bien definidas y documentadas en las prácticas operativas de la subestación. Por el contrario, las maniobras a realizar bajo circunstancias de falla en los sistemas de control y protección de una subestación, generalmente no se encuentran claramente definidas [3].

Para elaborar las estrategias de atención de fallas no destructivas en subestaciones, se debe analizar cada uno de los posibles escenarios de falla para determinar las causas y soluciones. El punto de partida para analizar los escenarios mencionados, es el estudio de las características de operación, de funcionamiento y la descripción de equipos de la subestación. Adicionalmente, se estudia el comportamiento de los equipos y sistemas en condiciones anormales y el funcionamiento de todo sistema ante la falla de alguno de sus componentes (efecto de fallas individuales en el comportamiento del sistema). Así mismo, se requiere establecer escenarios de falla, teniendo en cuenta los requerimientos y el nivel de especialización exigido por la circunstancia de falla.

En el caso de fallas destructivas de los equipos, se requieren acciones que involucran toda la organización. Para estos casos, se han desarrollado una serie de estrategias para la atención de transformadores, interruptores, seccionadores y salas de control y protección, elementos que son prioritarios para la seguridad, confiabilidad y continuidad en la prestación del servicio. Estas estrategias son los planes de contingencias asociados a los equipos, descritos metodológicamente en [2], [3] y [4], y se desarrollan para cada equipo en [5] y [6]

Cuando ocurren fallas no destructivas se requiere de la elaboración y aplicación de procedimientos encaminados a restablecer la disponibilidad del equipo o del sistema, finalizar maniobras inconclusas de los equipos primarios de potencia, localizar el punto de falla o realizar un diagnóstico que facilite la labor del personal calificado. Los procedimientos buscan aprovechar los recursos existentes en la subestación, tal como se presenta metodológicamente en [3], [4] y [7], y se implementa para cada subestación en [8], [9], $[10],[11],[12],[13]$ y [14].

\section{METODOLOGÍA}

El planteamiento metodológico utilizado para el desarrollo de las estrategias de restablecimiento de las subestaciones de transmisión, contiene tres etapas básicas: análisis, elaboración y validación. El esquema se presenta en la figura 1.

\section{a. Etapa de análisis}

En esta etapa, se propone un estudio sistemático de la naturaleza del problema incluyendo recopilación y análisis de la información, selección de datos estadísticos de fallas y recopilación de las experiencias del personal. Esta etapa consta de las siguientes actividades:

v Recopilación y análisis de información, consistente en el estudio inicial de aspectos conceptuales relacionados con los planes de contingencia para instalaciones eléctricas, el estudio de la reglamentación relacionada con el problema, y la recopilación y análisis de la información sobre los tipos de subestaciones y equipos existentes.

$\square$ Determinación de equipos y sistemas críticos, con base en el análisis de confiabilidad del funcionamiento de los equipos y su impacto en la disponibilidad en la operación de las subestaciones, tal como se presenta en [2][3][4]. De acuerdo con las referencias, es prioritaria la elaboración de las estrategias de fallas no destructivas para interruptores, seccionadores y sus circuitos de control; sistema de servicios auxiliares; equipos de protecciones; sistemas de medida; sistemas de registro de fallas $y$ sistemas de comunicación.

■ Elaboración de escenarios de falla a partir del 
análisis de las características de funcionamiento de los equipos, las estadísticas de falla y la experiencia del personal. Se deben definir las fallas más comunes y/o críticas para las cuales hay que elaborar los procedimientos de restablecimiento.

\section{b. Etapa de elaboración}

En esta etapa se hace el planteamiento de propuestas particulares para el plan de restablecimiento de cada equipo. Para su elaboración se desarrollan las actividades planteadas en la figura 2.

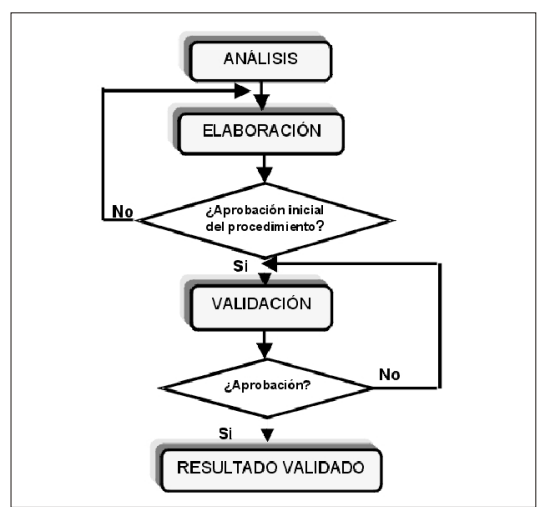

Figura 1: Metodología básica desarrollada para la elaboración e implantación de las estrategias de atención de fallas de subestaciones

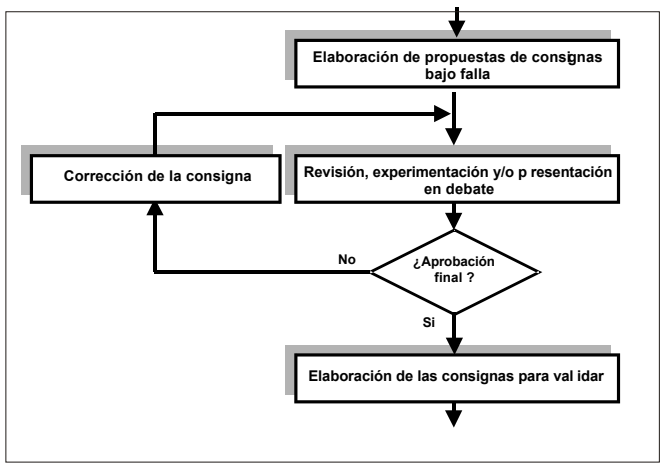

Figura 2: Metodología de elaboración de las estrategias de restablecimiento de subestaciones.

La elaboración de propuestas de consignas bajo falla se hace a partir de la definición de las fallas más comunes y/o críticas. Para el desarrollo de estos procedimientos (consignas), se utilizaron los planos de la subestación, los manuales de los equipos y la experiencia del personal. Las consignas contienen la descripción de las acciones a realizar en caso de falla no destructiva, evidenciada por la activación de una alarma, o la inadecuada operación de los equipos de protecciones, de control, de comunicaciones, de medida, de registro de fallas, de servicios auxiliares o de maniobras (falla en el cierre y/o apertura de seccionadores e interruptores).

El objetivo de la actividad de revisión y/o presentación a debate consiste en discutir la estrategia de atención de la falla no destructiva, bajo las condiciones particulares de ocurrencia. En esta fase, es de vital importancia la participación del personal de operación y los grupos de mantenimiento de las subestaciones. Realizada la revisión, se deben realizar las correcciones respectivas en la consigna para su aprobación final.

\section{c. Etapa de validación}

El proceso de validación tiene como objetivo el de revisar, actualizar y mejorar las consignas bajo falla, teniendo en cuenta las condiciones específicas de las subestaciones y los cambios introducidos en los circuitos de la subestación, así como detectar y corregir errores cometidos en la elaboración de los documentos de atención de las fallas no destructivas. Esta etapa permite además, capacitar al personal en el manejo y aplicación de los procedimientos de atención ante fallas no destructivas en subestaciones (consignas bajo falla).

Un mecanismo de validación es la realización de simulaciones de fallas para evaluar los procedimientos y el establecimiento de materiales de apoyo para reportar y registrar los resultados. Mediante la validación de las consignas, se aumenta la confiabilidad de los procesos redactados en ellas y se permite la incorporación del asistente de subestaciones al estudio y análisis de los procedimientos.

La validación comprende las actividades de análisis, verificación, simulación y corrección, tal como se presenta en la figura 3. 
La actividad de análisis consiste en la revisión de la consigna y la definición del plan que permita realizar las acciones propuestas, garantizar el cometido de la consigna bajo falla y evitar que se puedan presentar daños en los sistemas de la subestación. La revisión incluye el análisis de la subestación y su relación con la consigna. La definición del plan incluye la programación de las actividades de verificación y simulación, teniendo en cuenta la implicación que cada acción tiene en el funcionamiento de los equipos o del sistema.

La verificación busca corroborar la información contenida en las consignas, contrastándola con lo ocurrido en la simulación de fallas en cada subestación. En esta etapa se debe garantizar la concordancia entre la información del documento de atención de fallas con los sistemas que realmente hay en la subestación (conexiones, bornes, gabinetes, terminales, etc).

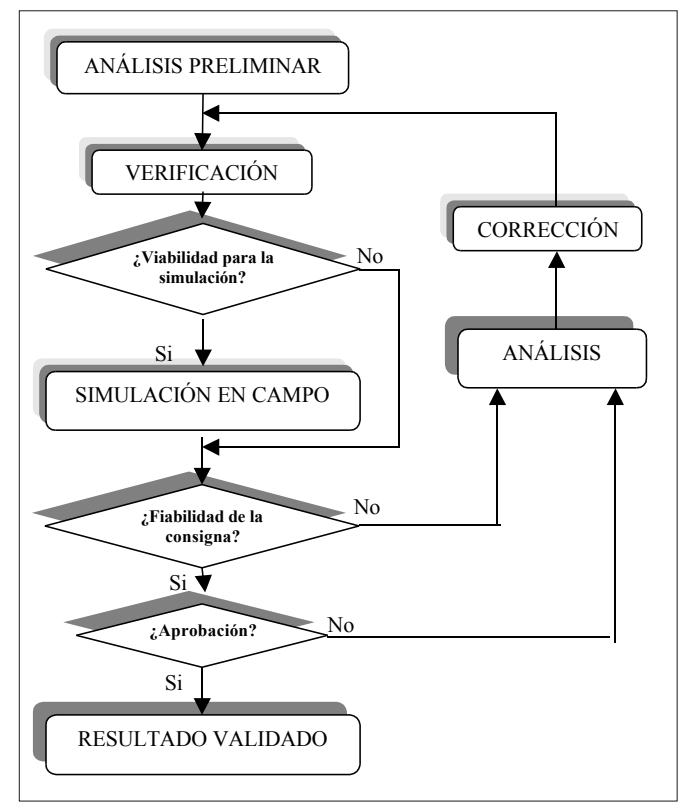

Figura 3: Proceso metodológico para la validación de las estrategias de fallas no destructivas en los equipos y sistemas de las subestaciones

La simulación consiste en poner a prueba los procedimientos establecidos en la consigna bajo falla. Para realizar la simulación se utilizan partes de la subestación que no estén en funcionamiento, donde se producen fallos intencionalmente, para que el operador, siguiendo la consigna, encuentre su causa y reponga el sistema. La simulación es el método ideal de validación ya que además de poner a prueba la consigna, prepara al asistente de la subestación en el empleo de las consignas bajo falla.

La corrección se realiza con la información de las fases de verificación y simulación. Se deben evaluar los resultados y revisar las causas de las falencias detectadas en las consignas, durante los procesos de verificación y simulación. Los resultados de la revisión se utilizan para ajustar la consigna bajo falla. Esta actividad comprende la definición de falencias de la consigna, verificación de la validez de las correcciones a través de discusiones técnicas de los cambios de la consigna y la aprobación final.

\section{DESARROLLO DE LAS CONSIGNAS BAJO FALLA}

La metodología de desarrollo permite obtener la estructura y el alcance de las consignas bajo falla. El alcance está limitado a las acciones permitidas, de tal forma que no se comprometa la integridad y estabilidad de los sistemas y equipos de la subestación y del sistema de transmisión.

Dentro de la estructura de una consigna bajo falla se destacan tres aspectos básicos: la descripción de la anomalía, la descripción de la posible causa y el procedimiento solución. Esto incluye desde el reconocimiento de las alarmas hasta la ejecución de acciones que conduzcan a determinar la permanencia en servicio del equipo fallado, o la revisión en planos del circuito de control básico de cierre/apertura.

Las consignas bajo falla son de dos tipos: consignas bajo falla que aparecen cuando se realiza una consigna operativa de equipos primarios de potencia o de servicios auxiliares; y consignas bajo falla asociadas a la aparición de alarmas relacionadas con los servicios auxiliares, el sistema de registro de fallas, el de medida, el de comunicaciones y con los equipos de protección y control. 


\section{a. Consignas bajo falla para atender fallas que se evidencian en el momento de la ejecución de una consigna operativa}

Estas consignas están diseñadas para atender fallas no destructivas en los equipos de maniobra (seccionadores/interruptores), y sus sistemas de control y protección. Corresponden a fallas que se evidencian por la ausencia de respuesta a las órdenes de cierre o apertura en mención, durante la realización de una maniobra operativa en la subestación.

Para la elaboración de las estrategias de acción ante fallas en la operación de los equipos primarios de potencia o equipos de maniobras de servicios auxiliares, es necesario realizar un análisis de las causas de la no operación de los mismos, así como el estudio de los equipos y sistemas.

En primer lugar, se debe identificar el diagrama funcional del sistema (equipo y sistema de control), y de la relación con los demás sistemas (configuración, protección, servicios auxiliares, etc.), para identificar las partes funcionales principales para el sistema y su interpretación. En la figura 4 se muestra el diagrama funcional de los interruptores y seccionadores y su relación con los sistemas control y protección. Como ejemplo de la interacción entre estos sistemas, en las figuras 5 y 6 se indica el circuito de control de un interruptor de $230 \mathrm{kV}$, de SF6 y un seccionador, de una subestación de transmisión.

En segundo lugar, se deben identificar o conocer las causas genéricas de las fallas que se presentan en la operación de los equipos, abstrayendo la complejidad de las acciones y orientándose a identificar qué función deja de realizarse, el bloque funcional con problemas, la forma y la causa de la falla y la repercusión en el sistema [3] [15].

Algunas de las causas por las cuales los seccionadores o los interruptores no cierran/abren, son: a) Las condiciones de operación no se cumplen, b)Se encuentra activada alguna alarma que enclava el equipo, c)El sistema de control se encuentra funcionando irregularmente, d)Problemas en los equipos que realizan la función de operación del seccionador o del interruptor, e)Problemas en los equipos de control de la subestación.

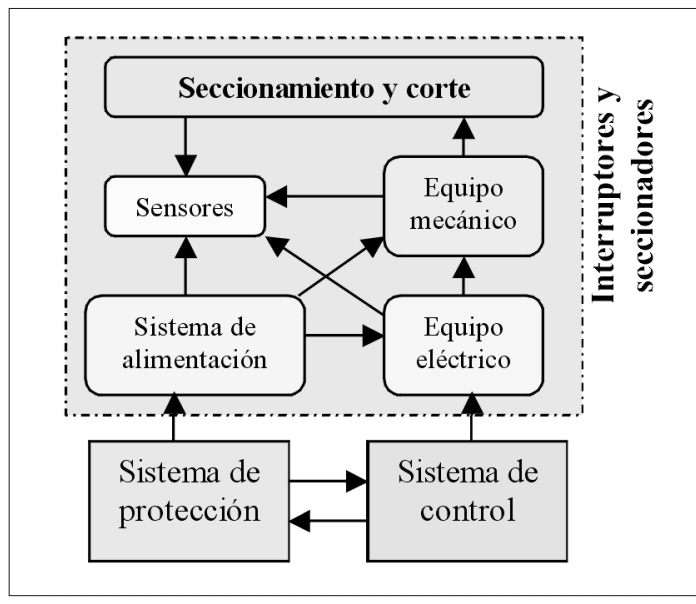

Figura 4. Esquema funcional de un interruptor y el sistema de control

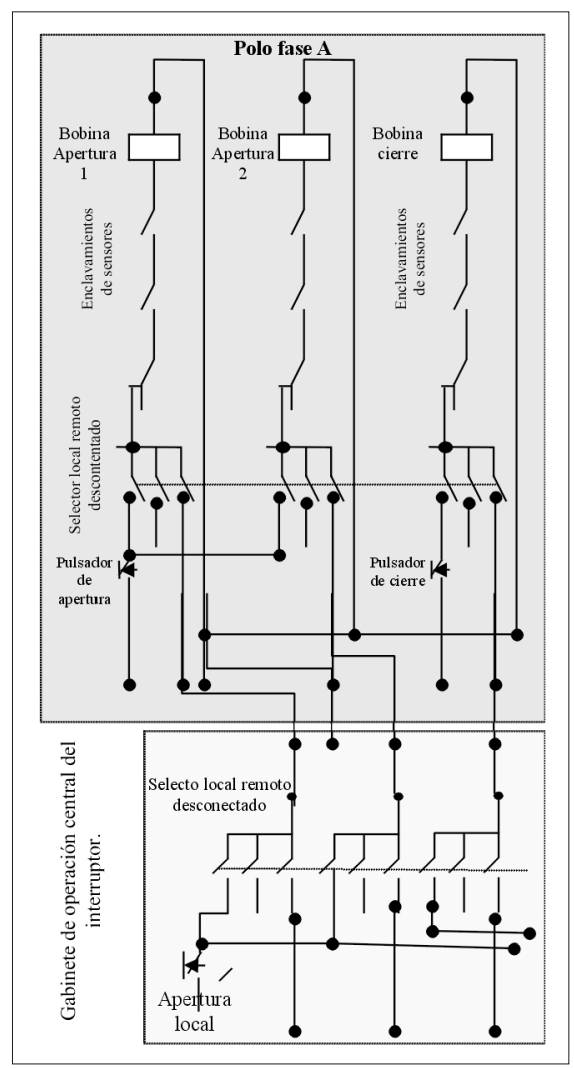

Figura 5. Circuitos de control de un interruptor Sf6 
En la figura 7 se presenta un esquema de los sistemas que acompañan los equipos de seccionamiento y corte, y además se presentan las causas que generan la falla o la inoperancia de los equipos primarios de potencia.

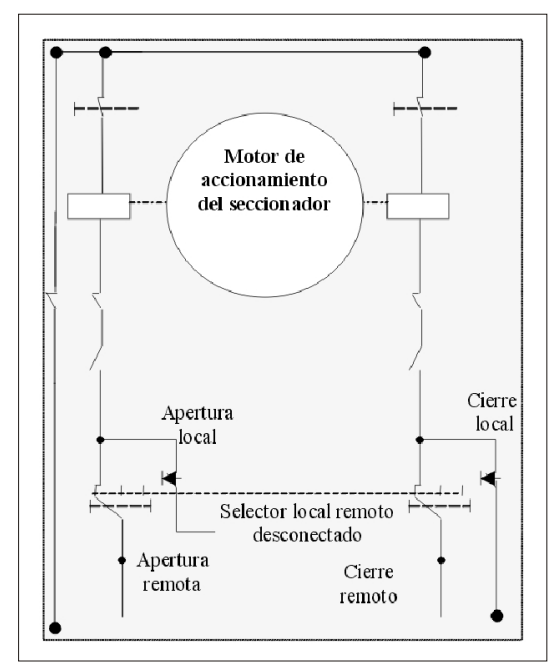

Figura 6. Circuito típico de control de un seccionador

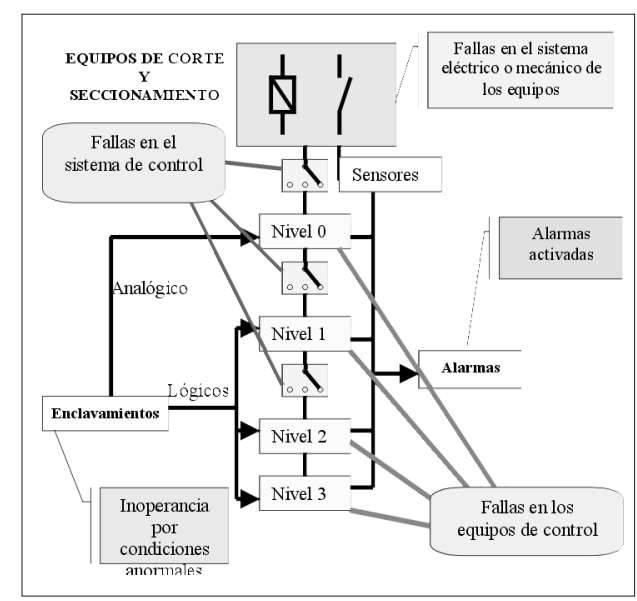

Figura 7. Fallas en equipos de seccionamiento y corte

Por último, la estructura propuesta en las consignas para la realización de las actividades, tiene un orden prioritario de acciones así: Revisión de condiciones iniciales, cambio de nivel de operación, cambio de consigna operativa (en los casos que sea posible), revisión posterior a la culminación de la consigna operativa y revisión en planos del circuito de control. El procedimiento está asociado a la maniobra operativa realizada, tal como se presenta en la figura 8.

En la mayoría de consignas bajo falla, y en caso de que no sea posible solucionar la falla siguiendo las instrucciones dadas, se sugiere hacer un seguimiento de la señal de tensión directamente en el circuito de control que se presenta como anexo de la consigna.

\section{b. Consignas bajo falla asociadas a la aparición de alarmas}

Cuando ocurre una alarma en la subestación, se enciende la señal luminosa en el anunciador y se energiza la alarma sonora. El asistente de la subestación realiza el reconocimiento de la alarma y a continuación sigue las acciones propuestas en las consignas bajo falla no destructiva. Luego se repone el anunciador de alarmas y se registra el evento en la bitácora de la subestación.

Las consignas bajo falla asociadas a la aparición de alarmas, ya sea en el panel indicador o en los propios equipos, están organizadas en un formato que permite reconocer causas y soluciones para la correcta reposición de la alarma o para la acción correctiva. Este tipo de consignas tienen una estructura básica de diseño que contiene: la identificación de señal de alarma, verificación de la veracidad de la señal y revisión de efectocausa, revisión del circuito de alarma en planos y por último realización de consigna operativa. La secuencia seguida en estas consignas se presenta en la figura 9.

Las consignas bajo falla asociadas a las alarmas están elaboradas para aquellas señales que tienen mayor importancia y para las cuales el asistente de operación puede tomar alguna acción correctiva. Éstas se dividen en tres partes que son: descripción de la anomalía, posibles causas y posibles soluciones.

Las alarmas típicas de una bahía de línea son: disparo protecciones principales o de protecciones de respaldo, disparo del relé falla interruptor, recepción y emisión de señal de 
teleprotección, baja presión de SF6, falla mecanismo de operación del interruptor, discrepancia de polos, falla circuitos de disparo, falla fusibles secundarios de transformador de tensión y falla alimentación de corriente continua [16], [17], [18] y [19].

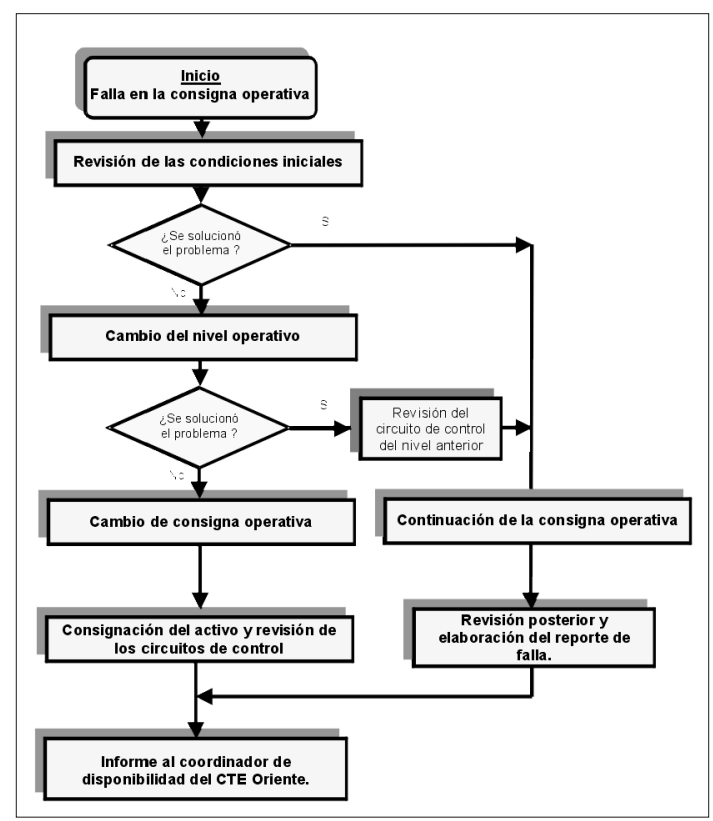

Figura 8. Procedimiento básico seguido en las consignas bajo falla no destructiva asociadas a consignas operativas de equipo de patio

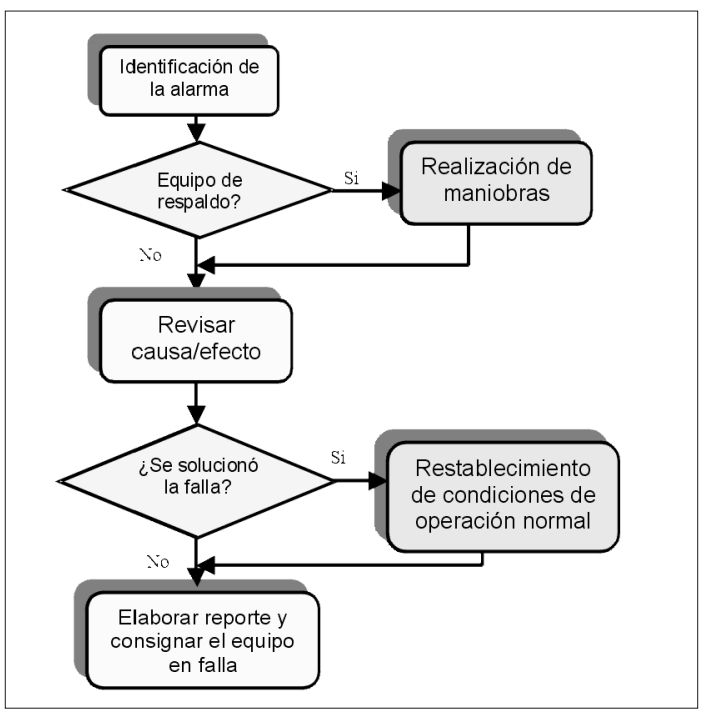

Figura 9: Procedimiento básico seguido en las consignas bajo falla asociadas a la aparición de alarmas en el panel indicador
Las alarmas típicas en un transformador de potencia son: operación protección diferencial, operación relé sobrecorriente (baja, alta tensión), operación relé Buchholtz, operación relé de presión de aceite, falla enfriamiento, alarma sobretemperatura, disparo por sobretemperatura, cambio derivación incompleto, falla regulador de tensión, falla circuitos de disparo, falla fusibles secundarios, falla alimentación corriente continua y discrepancia de polos [20].

En servicios auxiliares las alarmas típicas son: falla en barras, falla en baterías, falla en el cargador de baterías, falla en los alimentadores de corriente alterna, falla en los alimentadores de corriente continua, falla en el transformador de servicios auxiliares y falla en planta de emergencia [15] [21][22] y [23].

El sistema de registro de falla presenta alarmas de fallas, visualizadas en los páneles indicadores. Éstas son: falla alimentación y falla interna originada por falla del canal de comunicación; falla de comunicación entre el concentrador y alguna estación de adquisición de datos; falla de comunicación entre el concentrador y el registrador de fallas enrutador del sistema de adquisición de datos y falla en despliegue de las señales en proceso.

Los equipos de medida indican una falla interna a través de un panel indicador o a través de mensajes en la interfase de usuario del equipo. Las fallas más comunes son: Fallas en la alimentación del equipo, desactualización registro de tiempo del contador, alarma de nivel de batería baja, ausencia de lectura en la pantalla del contador, falla en las señales de tensión y corriente y falla del contador principal, entre otras.

Los equipos de comunicaciones presentan sistemas de supervisión de sus funciones que envían señales de alarmas al sistema de control de la subestación o indican las fallas en el panel frontal del equipo. Las alarmas pueden ser originadas por: falla en la alimentación del equipo, falla en el canal de voz, falla emisión en el equipo de teleprotección, falla en el hardware, lectura de 
de teleprotección, falla en el hardware, lectura de los mensajes de error del equipo y fallas internas en los módulos. En la figura 10 se muestra un esquema de flujo de información de señales de alarmas desde los equipos a los anunciadores (Panel o computadora).

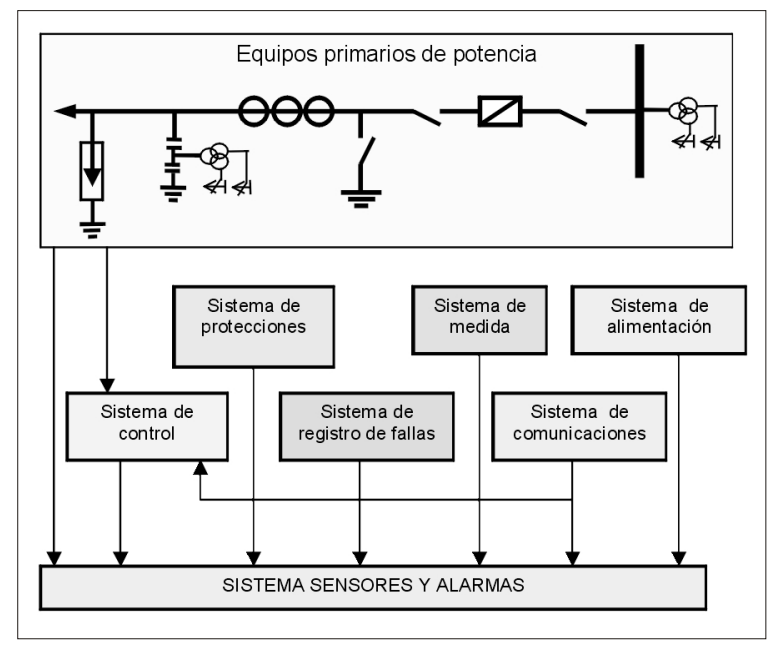

Figura 10: Esquema funcional del sistema de señalización y alarmas

\section{RESULTADOS}

La metodología de estrategias de atención de fallas descrita en este artículo, se aplicó a la elaboración de las consignas bajo falla de diez subestaciones en la zona atendida por el CTE Oriente de Interconexión Eléctrica S.A. conformando una base de datos de aproximadamente 2.600 consignas. En la actualidad, con esta información, se está terminando una investigación en asocio con la UIS y Colciencias, para desarrollar un sistema de simulación para la operación y atención de fallas en subestaciones de transmisión.

Para los equipos de control y protección se realizaron procedimientos guías para que el operador pueda intervenirlos en caso de falla, o de obtener la información sobre fallas en el sistema en caso de actuación de los mismos. En total se realizaron siete tipos de consignas bajo falla no destructiva para las diferentes clases de equipos en las subestaciones analizadas. En la tabla 1 se puede ver los resultados de las consignas de fallas elaboradas.

Tabla 1: Múmero y clase de consignas elaboradas

\begin{tabular}{|l|c|}
\hline \multicolumn{1}{|c|}{ Tipo de consigna } & Consignas Elaboradas \\
\hline Asociadas a la inoperancia de equipos de patio & 862 \\
Asociadas a servicios auxiliares & 186 \\
Asociadas a equipos de control y protecciones & 52 \\
Asociadas al sistema de comunicaciones & 180 \\
Asociadas a equipos de medida & 76 \\
Asociadas a equipos de registro de fallas & 82 \\
Asociadas a alarmas de los equipos primarios & \\
de potencia & 1163 \\
\hline Totales & 2601 \\
\hline
\end{tabular}

Cada uno de los tipos de consignas presentados en la tabla 1, están diseñadas para atender fallas en los subsistemas de la subestación de transmisión. Cada una de las consignas se encuentra disponible en la subestación para que sean usadas como guía de restablecimiento del servicio. En el caso particular de las consignas asociadas a alarmas, estas se deben usar cuando no se está desarrollando ninguna consigna operativa pero se activa la señalización de alarma en algún equipo de potencia.

\section{CONCLUSIONES}

■ Las consignas bajo falla elaboradas, sirven para atender las fallas no destructivas de los sistemas de maniobra, control y protección de las subestaciones de transmisión. El método se aplicó a la elaboración de las consignas bajo falla de diez subestaciones bajo responsabilidad del CTE Oriente de Interconexión Eléctrica S.A. conformando una base de datos de aproximadamente 2600 consignas.

■ La estructura de las consignas presenta los procedimientos a seguir cuando aparecen evidencias de fallas reparables en equipos o sistemas, o cuando equipos de maniobra, control y/o protección no responden a una orden de operación. Permite además, mejorar los índices de disponibilidad de equipos, mediante la reducción de los tiempos de corrección de fallas. 
$\checkmark \quad$ La aplicación de las consignas bajo falla, tiene como consecuencia la reducción del impacto de las situaciones de emergencia, el apoyo al cumplimiento de las metas de disponibilidad de activos; la mejora de la continuidad del servicio al usuario final y el aumento de la capacidad de los asistentes de la subestación.

घ En términos generales, los beneficios económicos de la implementación de las consignas bajo falla, están asociados al mejoramiento de la continuidad del servicio de transmisión, disminución en el tiempo empleado para la localización y diagnóstico de fallas, y la reducción del costo de mantenimiento asociado al desplazamiento de los técnicos del Grupo de Mantenimiento (GEM) a la subestación bajo contingencia.

$\square$ Las consignas bajo falla conforman una valiosa base de información. Mediante una adecuada representación del conocimiento se pueden desarrollar reglas de inferencia para conformar un sistema inteligente, que sirva de soporte en la atención de circunstancias de falla de subestaciones de transmisión. Usando esta información se propone el montaje de un sistema inteligente para brindar soporte en la atención de fallas en las subestaciones y para realizar módulos de entrenamiento para el personal de operación. Esto permite a su vez, el planteamiento de recomendaciones para el diseño de las futuras subestaciones eléctricas.

\section{BIBLIOGRAFÍA}

[1]Bollen M, "Understanding Power Quality problems" IEEE Press. Mew York. 2000.

[2]Mora J., Carrillo G., Jaimes J. "Strategies for the electric Supply Restoration in Colombia Transmission Substation". IEEE Power Tech. Bologna, Italy, June 2003.

[3]Mora J. J., Carrillo G. Desarrollo del plan de contingencia para equipos críticos principales y equipos de control y protección de subestaciones de transmisión, Tesis de grado. Universidad Industrial de Santander. Bucaramanga. 2001.
[4]Carrillo G., Mora J., Jaimes J., Estrategias para el restablecimiento del servicio en subestaciones de transmisión. Mundo eléctrico colombiano, vol 16 \#46 EneroMarzo. 2002.

[5]Álvarez E. D., Quintero J. H.. "Desarrollo del plan de contingencia para fallas destructivas de transformadores e interruptores de potencia de propiedad de Interconexión Eléctrica S.A. E.S.P. ISA CTE Oriente". Proyecto de pregrado de Ingeniería Eléctrica, dirigido por: G. Carrillo, J.J. Mora. UIS 2001

[6]Santos G., Beltrán E., Sequeda A. "Planes de contingencia para fallas destructivas en salas de control y protección de subestaciones de transmisión". Proyecto de pregrado de Ingeniería Eléctrica, dirigido por: G. Carrillo, J. Ariza. UIS 2002

[7]Carrillo G., Ariza J. "Validación y complementación de los planes de contingencia", Informe de avance del proyecto de investigación, correspondiente al $50 \%$. ISACTE Oriente, 2001

[8]Álvarez C. E. "Desarrollo del plan de contingencia para equipo de la subestación San Mateo propiedad de Interconexión Eléctrica S.A. E.S.P. ISA CTE Oriente". Proyecto de pregrado de Ingeniería Eléctrica, dirigido por: G. Carrillo, J. Mora. UIS 2001

[9]Antelíz R. "Desarrollo del plan de contingencia para equipo de la subestación Ocaña propiedad de Interconexión Eléctrica S.A. E.S.P. ISA CTE Oriente". Proyecto de pregrado de Ingeniería Eléctrica, dirigido por: G. Carrillo, J.J. Mora. UIS 2001.

[10] Ariza J. O.; Santamaría L. A. . "Desarrollo del plan de contingencia para equipo de la subestación Guatiguará propiedad de Interconexión Eléctrica S.A. E.S.P. ISA CTE Oriente". Proyecto de pregrado de Ingeniería Eléctrica, dirigido por: G. Carrillo, J.J. Mora. UIS 2001.

[11]Ferreira C. "Desarrollo del plan de contingencia para equipo de las subestaciones Toledo y Samoré propiedad de Interconexión Eléctrica S.A. E.S.P. ISA CTE 
CTE Oriente". Proyecto de pregrado de Ingeniería Eléctrica, dirigido por: G. Carrillo, J.J. Mora. UIS 2001.

[12] Gutiérrez L.; Miño J. "Desarrollo del plan de contingencia para equipo de las subestaciones Los Palos, Banadía y Caño Limón, propiedad de Interconexión Eléctrica S.A. E.S.P. ISA CTE Oriente". Proyecto de pregrado de Ingeniería Eléctrica, dirigido por: G. Carrillo, J.J. Mora. UIS 2001.

[13] Lara L.. "Desarrollo del plan de contingencia para equipo de la subestación Comuneros propiedad de Interconexión Eléctrica S.A. E.S.P. ISA CTE Oriente". Proyecto de pregrado de Ingeniería Eléctrica, dirigido por: G. Carrillo, J.J. Mora. UIS 2001

[14] Leiva Y. A.; Jaimes J. P. "Desarrollo del plan de contingencia para equipo de la subestación Primavera propiedad de Interconexión Eléctrica S.A. E.S.P. ISA CTE Oriente". Proyecto de pregrado de Ingeniería Eléctrica, dirigido por: G. Carrillo, J.J. Mora. UIS 2001.

[15] Carrillo G., Ariza J.. Informe técnico de avance proyecto "Sistema de simulación para la operación y la atención de fallas de subestaciones de transmisión". Bucaramanga, Marzo de 2003.

[16]ABB, Asea Brown Bovery Ltda. Metwork Partner AB. Manual de operación y mantenimiento del terminal de control REL 511 y 561.1994.

[17] ABB. Manual de Operación y Mantenimiento, Equipo de patio. Subestación Comuneros $230 \mathrm{kV}$. Folder A-100

[18] AEG. Manual de operación y mantenimiento, protección de línea 1 EPAC 3000 y Optimho. Subestación Primavera 230 KV, 1996. Volumen 07 y 0.8

[19]Commisioning and maintenance guide. Protection and automatic control equipment EPAC 3000. Agosto de 1995.

[20] Ramirez C. Subestaciones de Alta y Extra Alta tensión, Editor Mejía Villegas S.A, Segunda edición, 2004.
[21] Carrillo G., Ariza J. Propuesta de investigación "Sistema de simulación para la operación y la atención de fallas de subestaciones de transmisión" Bucaramanga, Mayo de 2002.

[22] Carrillo G.; Mora J. Desarrollo del plan de contingencia para equipos críticos principales y equipos de subestaciones, Informe 2000

[23] ISA-CTE Oriente. Guía para la operación y mantenimiento de la planta diesel. Subestación Comuneros. Bucaramanga, Abril de 2000. 